\title{
The glomerular code for odor representation is species specific in the honeybee Apis mellifera
}

\author{
C. Giovanni Galizia, SilkeSachse, Angelika Rappert and Randolf M enzel
}

Institut für Neurobiologie, FreieUniversitat Berlin, Konigin-Luise-Str. 28-30 14195 Berlin, Germany

Correspondence should beaddressed to C.G.G. (galizia@zedat.fu-berlin.de)

\begin{abstract}
Odors are coded by glomerular activity patterns in the insect antennal lobe (AL) and in the mammalian olfactory bulb. We measured glomerular responses to 30 different odors in the AL of honeybees using calcium-sensitive dyes. By subsequently staining glomeruli and identifying individual glomerular outlines, we were able to compare the patterns between animals. Regardless of whether the odors were mixtures or pure substances, environmental odors or pheromones, their representations were highly conserved among individuals. Therefore, it may be possible to create a functional atlas of the AL in which particular molecular receptive ranges are attributed to each glomerulus.
\end{abstract}

Physiological experiments have shown that odors evoke spatially organized activity patterns in both the vertebrate olfactory bulb ${ }^{1-6}$ and the insect antennal lobe ${ }^{7-12}$. Evidence is mounting that this spatial organization is conserved within a species: in rodents, axons of receptor neurons expressing the same receptor gene converge to equivalent positions in different individuals ${ }^{13}$. Physiological responses to particular odors also show similar species-typical activity patterns ${ }^{14}$; this was demonstrated using electrophysiological recordings in rabbits ${ }^{15}$, radioactively labelled 2-deoxyglucose in rats $^{3,4}$ and optical recording in zebra fish ${ }^{5}$. However, up to now, mapping activity patterns to morphologically identified glomeruli was possible only in pheromone processing systems 6,16 .

We show that activity patterns are conserved between individuals at the level of identified, 'ordinary' glomeruli in the AL of the honeybee. Such a dedicated role of glomeruli previously has been shown only for the macroglomerular complex in insects ${ }^{17}$ and the modified glomerular complex in mammals ${ }^{16}$. The AL of the worker honeybee consists of 156-166 glomeruli arranged peripherally in a single layer. The glomeruli possess stereotypical, species-specific morphology ${ }^{18}$. They range in diameter between 30 and $50 \mu \mathrm{m}$ and differ in shape and relative arrangement. This has made it possibleto createa three-dimensional atlas of the honeybee $A L 19,20$, in which each glomerulus is given a namecomposed of its afferent receptor axon tract ( $\mathrm{T} 1$ to T4) and a number (for example, T1-45 or T3-45).

\section{RESULTS}

To physiologically characterize identified glomeruli in an in-vivo preparation of the honeybee $\mathrm{AL}$, we monitored intracellular calcium with Calcium Green ${ }^{9,10}$. Previous measurements showed that elicited activity patterns are odor-specific, stable for repeated stimuli ${ }^{9}$ and bilaterally symmetrical ${ }^{11}$. When the $A L$ is stained with Calcium Green, glomerular borders cannot be anatomically identified (Fig. 1a). Therefore, to visualize the glomerular layout, we stained the AL with the membrane-soluble dye RH 795 fol- lowing physiological measurements (Fig. 1b). Wethen identified individual glomeruli using the AL atlas (www.neurobiologie.fuberlin.de/honeybeeALatlas ${ }^{20}$ ). This allowed us to create a map of identified glomeruli for each bee tested (Fig. 1c). Activity patterns imaged with the calcium dye were then superimposed onto the map of identified glomeruli of the AL (Fig. 1d). Because the $A L$ was removed for RH 795 staining, accessory structures (tracheae, for example, marked with arrows in Fig. 1d) were used as landmarks for correct positioning of the mask. With this procedure, measured responses to each odor tested were attributed to each morphologically identified glomerulus. For example, octanol elicited the strongest response in glomeruli 33 and 17 and weaker responses in 28, 52 and 60 of T1, as well as in T3-45 (Fig. 1e). Note that the optical projection of $A L$ combining several focal levels differs slightly from the projection obtained by the $20 x$ objective used in the physiological measurements at only one focal plane. This mismatch can be seen in glomerulus T1-17, for instance; because part of the glomerulus is out of focus, activity seems relegated to only one part of the glomerulus (Fig. 1e).

The activity elicited by odors was thus compared among individuals at the level of homologous glomeruli. For example, patterns of response to 1-hexanol appear to be conserved among the 21 individuals measured (Fig. 2): 1-hexanol elicits the strongest responsein glomerulus T1-28 in 17 of 21 individuals and weaker, less consistent activity in glomeruli T1-38 and T1-52.

How similar are these patterns between individuals? To statistically address this question, we applied a multivariate discriminant analysis to the normalized data (Table 1). Discriminant analysis allowed us to judge whether odors clustered in multidimensional space (where each dimension represents a glomerulus). If individual observations for two odors had representations that overlapped in multidimensional space, then the discriminant analysis would be unable to discriminate between the two odors and, hence, unable to determine the correct odor from the measured response. We found that most measurements $(86 \%)$ could be attributed unam- 
Fig. 1. Mapping identified glomeruli of the honeybee $A L$ by calcium imaging. (a) View of the AL stained with Calcium Green $A M$, as seen in the experiment. N ote poor contrast. 0 bjective, 20x; scale bar, $50 \mu \mathrm{m}$. (b) After calcium measurements, the tissue was stained with RH 795, revealing glomerular boundaries. 0 bjective, 40x; scale bar, $50 \mu \mathrm{m}$. (c) Reconstruction of the glomerular map from views as in (b) at different focal planes and using a 20x objective. Each glomerulus is represented by a unique, arbitrary color. The two green glomeruli at the bottom right could not be reliably identified. All glomeruli without tract labels are innervated by T1. (d) View of the AL as in (a) with a superimposed, reconstructed glomerular map. 0 bjective, 20x; scale bar, $50 \mu \mathrm{m}$. (e) False-color-coded spatial maps for responses of an individual to 1-octanol. Red indicates strongest response, blue, weakest (false-color bar is to the right). The reconstructed glomerular map of this individual is superimposed onto the response map. Compare with Fig. 1c for glomerular identity and with Fig. 3a. 0 bjective, 20x ; scale bar, $50 \mu \mathrm{m}$. (f) Time traces of the response of individual glomeruli (T1-17, T1-28, T1-33, T1-35) to 1-octanol, plotted in $\Delta \mathrm{F} / \mathrm{F}$ over time after correction for bleaching. Apparent increase toward zero before stimulus onset is a correction artifact (see Methods). W hite bar indicates the stimulus.

bigously to the correct odor. The mistakes are also telling: although most ( $90 \%$ ) patterns evoked by 1octanol (OC1) were cor rectly identified, one measurement was attributed to 2-octanol (OC2) and one to n-octane (OCT), chemically similar substances. The odor that evoked the worst performance was linalool (LIO), which was mistaken once for citral (CIT), once for lime blossom (LND) and once for orange (ORG). Indeed, linalool evoked a very diffuse pattern of activity (Fig. 4) with only limited enhancement in glomeruli T1-17 (shared with lime blossom), T1-30 (shared with orange) and T1-48 (shared with citral and orange).

Averaged response patterns of 24 bees to 30 odors were used to estimate the glomerular representation of each odor in Apis mellifera. (M ost bees were tested with a subset of odors; see M ethods.) We show the spatial position of individual activated glomeruli for the odors 1-octanol (Fig. 3b) and clove oil (CLV; Fig. 3c), as well as the pattern of glomeruli activated by each odor tested (Fig. 4). Of 34 entries with strong responses, 17 had a s.d. $<15 \%, 13$ between $15 \%$ and $25 \%$ and 4 between $25 \%$ and $35 \%$. No entries with variability greater than $55 \%$ were found. An illustrative example of this predictability is given: 2-octanone $(\mathrm{OCN})$ consistently elicited strong activation in glomerulus T1-33 (black color, average maximum response of 10.03 from 11 individuals), with responses in T1-28 that varied from equal to weaker than those of T1-33 (same circle size, but blue color). Less-active components of the pattern were T1-17, T1-29, T1-36, T 1-38, T1-39, T1-48, T1-52 and T3-45. There were weak responses in all glomeruli. In contrast, pentanoic acid (PES) gave much weaker responses (average maximum response 1.51) in glomerulus T1-21 of all of six individuals tested (black) and in T1-8 in most individuals (blue); a weak response was measured from T1-12. However, this glomerulus could only be identified in one animal (white circle). No responses above noise were measured in the other glomeruli (no symbol).
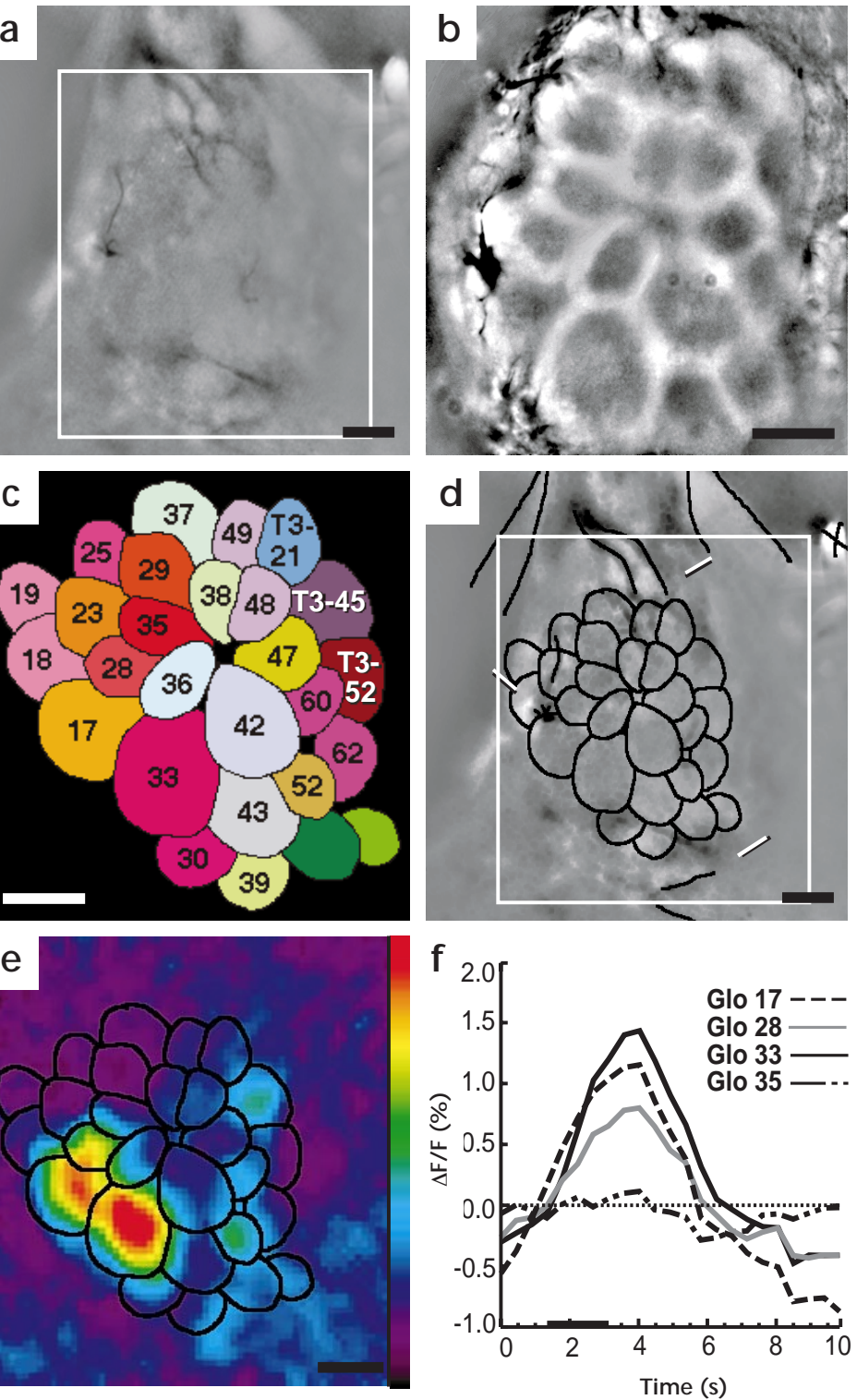

\section{Discussion}

This study showed that associations of individual glomeruli with characteristic molecular receptive ranges are conserved among conspecific individuals. Thus, odors are represented by characteristic, species-specific mosaics of activated glomeruli.

We have measured a substantial part of the honeybee olfactory code (Fig. 4). The complete code is certainly more complex: first, of a total of 160 glomeruli, only 38 could be imaged so far (24\%). Interestingly, T1 innervates 34 of these, almost $50 \%$ of the 70 glomeruli of this part of the AL. Second, dynamic aspects such as the sequence of glomerular activation $^{9,10}$ or fast temporal components ${ }^{21}$ are likely to be part of the complete olfactory code as well. In this study, glomeruli were each assigned an overall activity measure based on slow calcium measurements, thus ignoring any temporal features of the code. Third, each odor was consistently delivered at the same, non-saturating stimulus concentration routinely used in behavioral assays. Previously, we showed that elicited activity patterns are stable over a concentration range of two orders of magnitude ${ }^{9}$. In preliminary studies, however, we have found 
Fig. 2. Intraspecific variability of the response to 1-hexanol: comparison of response patterns to 1-hexanol in 21 individuals. Each row (A-U) represents responses of one individual, normalized to the maximum response. The maximum response is given in the right column $\left(R_{\text {int }}\right)$. Percent maximum responses are grouped into five bins of equal width, denoted by circle diameter (see legend). The subset of glomeruli shown represents $12 \%$ of the entire AL. G lomeruli that could not be identified in an individual are marked with a gray box. Missing entries denote glomeruli with below-noise responses. In almost all individuals, 1-hexanol is predominantly coded in glomerulus T1-28; only in individuals C, F, $\mathrm{G}$ and $\mathrm{H}$ was $\mathrm{T} 1-28$ not the strongest responder. Compare these results with the overall average ( $\mathrm{HX} 1$ in Fig. 4).

that patterns may change at even lower concentrations. Thus, the olfactory code presented here holds only for the concentrations tested (Fig. 4). Indeed, bees can discriminate among different odor concentrations ${ }^{22,23}$.

M ost odors elicited patterns made up of a few, strongly activated glomeruli. For example, isoamyl acetate (a component of the alarm pheromone) is coded in glomeruli T1-29, T1-38 and T1-48. In the one animal in which T1-82 could be identified, the glomerulus was strongly activated by isoamyl acetate. Orange (a complex plant extract) activates T 1-48 together with T 1-30, whereas T 1-48 and T3-45 are activated by limonene ( $a$ chemically pure substance; see Fig. $\mathbf{4}$ for other response patterns). M ost odors also weakly activate the entire AL; this background activity may be due to interneurons that branch to multipleglomeruli. Although no distinct patterns of activation were seen in response to the odors hexanoic acid, heptanoic acid, octanoic acid, nonanoic acid and n-decane, they too elicited a weak and below-noise maximal response, reflected in $R_{\text {int }}$. These odors may be coded in regions of the $A L$ that were outside the focal plane of our recordings. Responses to $\mathrm{CO}_{2}$ and 'queen substance' (queen pheromone) were very weak, making their interpretation unreliable. Q ueen substance is used both to attract male bees (drones) for mating and to inhibit sexual development in worker bees; it should be noted that worker bees- unlike drones-lack a macroglomerular complex.

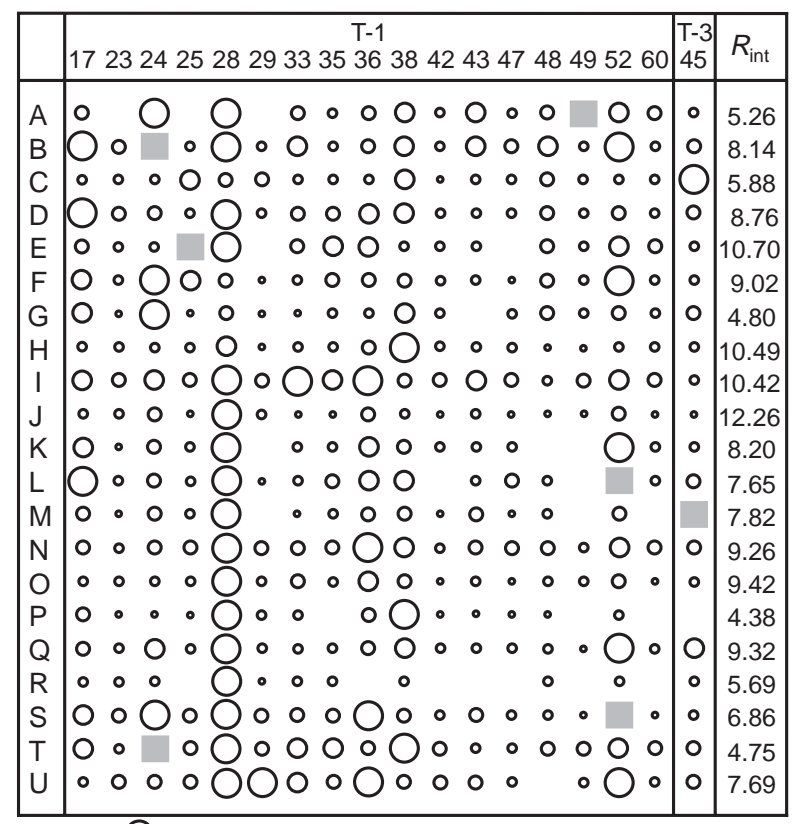

response: O80-100\%; O 60-80\%;040-60\%;॰20-40\%; • noise-20\%

The discriminant analysis indicates that the glomerular activity patterns are conserved among individuals. Furthermore, because a mere 18 glomeruli $(11 \%$ of the 160 glomeruli in honeybees) are sufficient to correctly identify most stimuli, we conclude that the coding of odors is redundant in addition to being species specific, multiglomerular and robust. Indeed, rats with partial bulbectomy are able to recognize odors with only $21 \%$ of their olfactory bulb intact 24,25 . Because afferent receptor cells contribute most to the measured signals ${ }^{11}$, the consistency of patterns between individuals implies that innervation of homologous glomeruli is similar for different individuals, and therefore must be genetically determined.
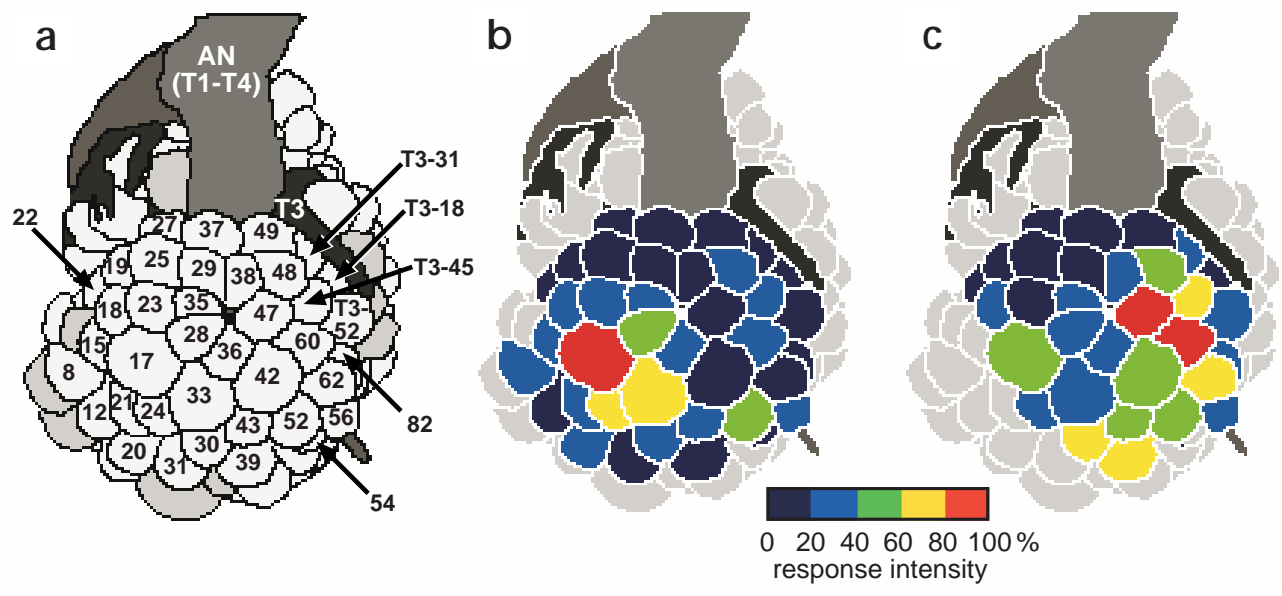

Fig. 3. A veraged glomerular spatial response patterns to 1-octanol and to clove oil. (a) Schematic view of the AL with the 38 most frequently recognized glomeruli labeled. AN, antennal nerve. 0 ther glo meruli recognized in only a few individuals are shown in gray; glomeruli in white without a label could not be recognized in the physiological measurements. (b) Graphic representation of the glomerular activity pattern as averaged from 21 individuals responding to 1-octanol. The color code gives the relative response intensity in five bins, corresponding to the circle sizes in Figs. 2 and 4. Glomeruli in gray could not be mapped reliably. (c) Glomerular activity pattern averaged from five individuals responding to clove oil. Color code, same as in (b). N ote that because of the smaller $n$, fewer glomeruli have been identified than for 1-octanol . 


\begin{tabular}{|c|c|c|c|c|c|}
\hline 0 dor tested & $\mathrm{n}$ & N umber correct & Percent correct & Misplaced odor & Mistaken \\
\hline ISO & 4 & 4 & $100 \%$ & - & - \\
\hline CIT & 4 & 3 & $75 \%$ & OCT & LIO \\
\hline GER & 9 & 7 & $77.8 \%$ & OCT, LIO & - \\
\hline CIN & 4 & 3 & $75 \%$ & PFM & \\
\hline EUG & 4 & 4 & $100 \%$ & - & - \\
\hline $\mathrm{ClO}$ & 3 & 3 & $100 \%$ & - & GER, OCT \\
\hline MNT & 3 & 3 & $100 \%$ & - & - \\
\hline LIM & 4 & 4 & $100 \%$ & - & - \\
\hline LIO & 7 & 4 & $57.1 \%$ & CIT, LN D, O RG & - \\
\hline HXI & 21 & 18 & $85.7 \%$ & $O C 2(2), O C N$ & $0 \subset 2$ \\
\hline $0 \subset 1$ & 21 & 19 & $90.5 \%$ & OC2, OCT & $0 \subset 2(3)$ \\
\hline $0 \subset 2$ & 18 & 14 & $77.8 \%$ & $\mathrm{HX} 1,0 \mathrm{C} 1(3)$ & $\mathrm{HX} 1(2), \mathrm{OC} 1$ \\
\hline$O C A$ & 10 & 10 & $100 \%$ & - & OCN \\
\hline OCT & 9 & 7 & $77.8 \%$ & CIO, CLV & CIT, GER, OC 1 \\
\hline CLV & 5 & 5 & $100 \%$ & - & OCT \\
\hline LND & 3 & 3 & $100 \%$ & - & LIO \\
\hline PFM & 4 & 4 & $100 \%$ & - & CIN \\
\hline ORG & 4 & 3 & $75 \%$ & LIM & LIO \\
\hline
\end{tabular}

Discriminant analysis of the glomerular activity patterns elicited from all measured individuals. The first column (odor) gives the odor tested (see Methods for abbreviations); $n$, number of animals tested; number correct, measurements correctly attributed to test odor: percent correct, percentage of measurements correctly attributed; misplaced odor, odors to which measured activity patterns were wrongly attributed-if more than one, the number of instances is indicated in brackets; mistaken odor, instances in which patterns were wrongly attributed to this odor. (For example, the row ' $\mathrm{ClO}$ ' shows that all three measurements of $\mathrm{CIO}$ have been attributed to $\mathrm{CIO}$ by the analysis; however, one GER and one O CT measurement have also been mistaken for CIO .) nurse bee) of the individual26,27. Therefore, in addition to dedicated molecular receptive ranges for each glomerulus, it seems that development and experience may modulate responses. Because the measured responses comprise components from all cells present in the AL, signal variability may arise from interneurons rather than receptor neurons. We cannot exclude the possibility that the variable components may depend on season of observation, ethotype or genetic background of individual bees.

Identified neurons and known, hard-wired neuronal circuits have long been established among insects ${ }^{28}$. However, stereotypical spatial organization of the olfactory bulb and conserved spatial activity patterns may also be a property of the olfactory bulb in mammals $s^{3,4,13}$, suggesting that homologous glomeruli may have identical molecular receptive ranges among mammals, as well. It is possible that olfactory maps optimize connection patterns between individual, active glomeruli. Indeed, measurements from neighboring mitral cells have shown similar receptive range profiles and response sharp-

Previously, we compared activity patterns without matching these patterns to the structures that generate them. In our first study ${ }^{9}$, we argued that the representation of pheromones (citral) was conserved between individuals whereas floral odors (for instance, hexanol) weremorevariable. Weshow herethat therepresentations are equivalent for all odors, irrespective of their functional roles. Citral elicited a strong response in the medially positioned glomerulus T3-45 (Fig. 4), whereas the pattern for hexanol, involving a series of weaker glomerular responses, was more complex, changing in appearance with the orientation of the antennal lobe. Such differences between individuals may only be resolved by mapping the responses to morphologically identified glomeruli. Still, some variability overlay stereotypical glomerular activity patterns (Fig. 2). Several experimental constraints may have reduced pattern similarity between individuals, including errors in the glomerular borders introduced by projection artifacts (Fig. $\mathbf{1 d}$ ), misidentification of glomeruli, spoiled single measurements due to movement artifacts, differences in relative responses between animals due to variable focal plane placement, poor signal-to-noise ratio resulting from low signal amplitude or minute differences in stimulus timing. Nevertheless, we believe a part of the observed variability to bea genuine consequence of biologically relevant processes. Genetic variation could, for example, result in structural variability between animals. Furthermore, there are plastic connections in addition to hard-wired connectivity in the AL. Indeed, calciumactivity patterns in the AL have been shown to changeafter appetitive training in the honeybee ${ }^{12}$. Also, glomerular size has been shown to be plastic, and the relative sizes of individual glomeruli to be reversibly dependent on the behavioral role (forager or ening due to lateral inhibition from neighboring glomeruli29. We are currently investigating interactions between active glomeruli in honeybees.

\section{Methods}

Measurement of odor-evoked activity patterns. In-vivo cal cium recordings were done as described ${ }^{11}$. All measurements were done between January and $M$ arch, 1998. Bees came from four different hives kept in a flight chamber on a constant night/day cycle. After capture, bees were quickly anesthetized by chilling and placed in a plexiglass chamber. The head capsule was then opened and the brain exposed and floated in a solution of Calcium Green 1 or 2 AM (M olecular Probes, Eugene; $50 \mu \mathrm{g}$ dye was first dissolved in $50 \mu \mathrm{l}$ Pluronic in DM SO and then diluted in $950 \mu \mathrm{l}$ Ringer's saline: $130 \mathrm{mM} \mathrm{NaCl}, 6 \mathrm{mM} \mathrm{KCl}, 4 \mathrm{mM} \mathrm{M} \mathrm{gCl} 2,5 \mathrm{mM} \mathrm{CaCl}$, $160 \mathrm{mM}$ sucrose, $25 \mathrm{mM}$ glucose, $10 \mathrm{mM}$ HEPES, pH 6.7, $500 \mathrm{mOsmol}$ ). After staining for $1 \mathrm{~h}$, the brain was rinsed in fresh Ringer's and the sion with Ringer's at room temperature ( $1 \mathrm{ml}$ per min; $22^{\circ} \mathrm{C}$ ). At all stages, great care was taken not to wet the antennae. Images were taken at a rate of $\sim 2$ images per second with 240 ms exposure time per image, and measured with a 12-bit CCD camera (Photometrics CH 250A, Tucson, Arizona). The data were analyzed on a M acintosh computer with custom software using IDL (Research Systems, Colorado). Signals were calculated as relative changes in fluorescence $(\Delta F / F)$. First, the resulting time-courses were linearly corrected against bleaching, and then the control air stimulus was subtracted. Because bleaching follows an exponential function, this procedure leads to the apparent artifactual increase of signal from negative toward zero before the stimulus onset.

For all odors, $4 \mu \mathrm{l}$ of the pure liquid substance was placed on filter paper $\left(1 \mathrm{~cm}^{2}\right)$, and thefilter placed in a 1-ml plastic syringe. Stimuli were delivered manually by puffing $0.8 \mathrm{ml}$ of odor-saturated air into a constant air stream directed to the animals' antennae. Stimulus timing was recording chamber placed under the microscope under constant perfu- 


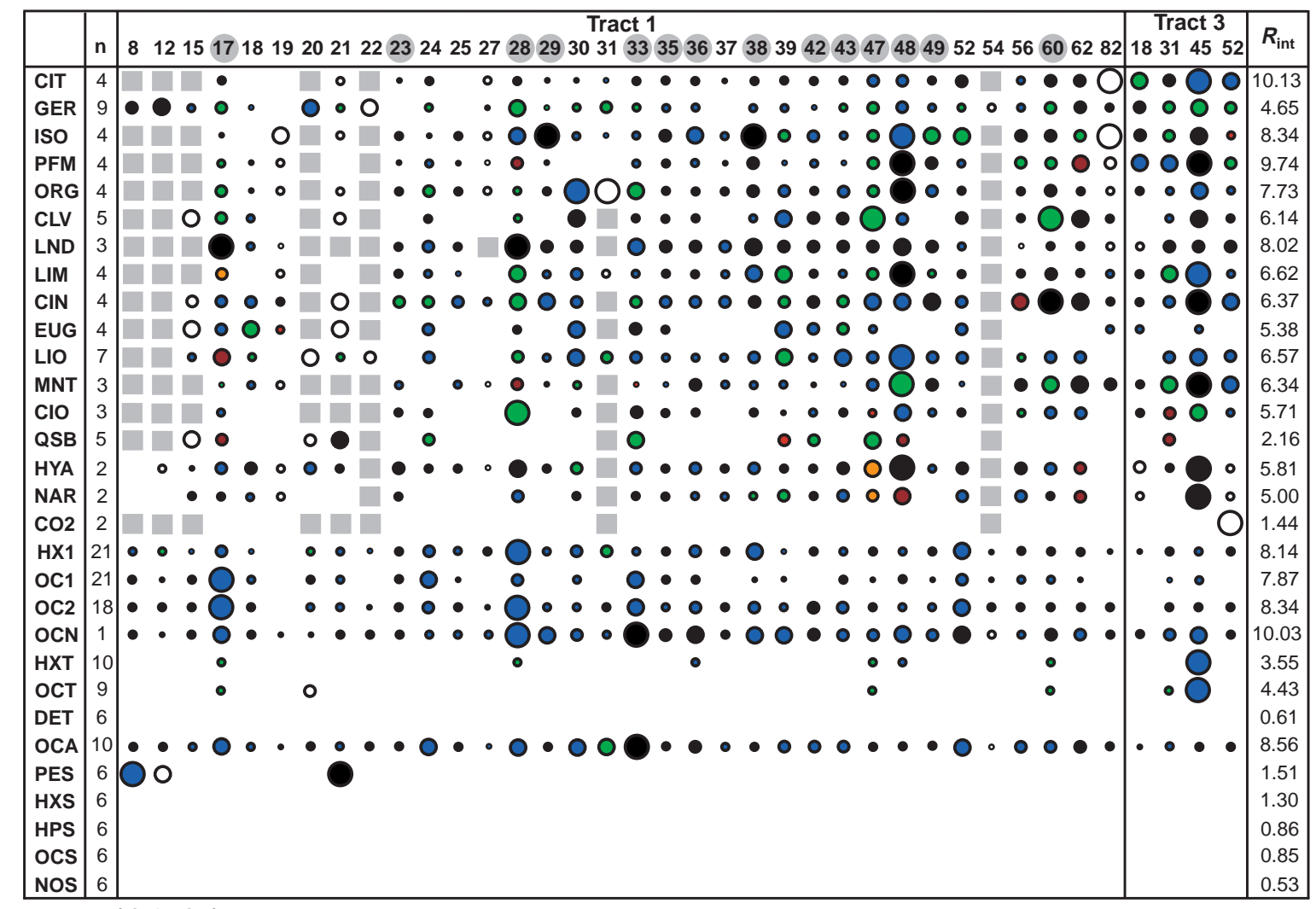

response (circle size):

Consistency range (color; white circle: $n=1$ )

O $80-100 \%$;O60-80\%; O40-60\%;• 20-40\%; •noise-20\%; <noise; no measurement s.d.<15\%; s.d.<25\%; s.d.<35\%; s.d.<45\%; s.d.<55\%

Fig. 4. Glomerular representation of all odors tested. Each row represents one odor, each column, an identified glomerulus (see Methods for odor abbreviation). Each entry is the median response of several individuals (compare row HX 1 with Fig. 2, and row 0 C 1 and CLV with Fig. 3). The number of measurements varies for both odor ( $n$ given in the column to the left) and glomerulus, because not all glomeruli could be identified in all animals. G lomeruli marked in the title line were identified in all. Response intensity is coded by circle size. Missing entries denote below-noise responses. Circle color indicates the consistency (s.d.) for the single measurements across individuals; white indicates an $n$ of one. The right column gives the averaged maximum response $R_{\text {int }}$.

given by computer-controlled sound cues. Each measurement block consisted of one control stimulus (air and filter paper without odor) and four odorant stimuli, with an interstimulus interval of $45 \mathrm{~s}$. In each bee, responses were measured for 10-20 such blocks.

Odors used. All odors without source reference were from SigmaAldrich, Deisenhofen. See Fig. 4 for the number of animals tested with each odor. CIT, citral; GER, geraniol; ISO, isoamyl acetate; PFM , peppermint oil (local drugstore); ORG, orange oil (local drugstore); CLV, clove oil (local drugstore); LND, lime blossom (Tilia, local drugstore); LIM , limonene; CIN , cineol; EUG, eugenol; LIO (+)-linalool; M NT (-)-menthol diluted in $\mathrm{n}$-hexane; CIO, DL-citronellol; QSB (queen substance), synthetic queen-bee mandibular complex (Phero tech, Delta, British Columbia); HYA, headspace of hyacinth flower, extracted in the lab and dissolved in $n$-hexane. NAR, headspace of narcissus flower (extracted as $\mathrm{HYA}$ ); $\mathrm{CO}_{2}$, carbon dioxide; $\mathrm{HX}$ 1, 1-hexanol; $\mathrm{OCl}$, 1-octanol; OC2, 2-octanol; OCN; 2-octanone; HXT, n-hexane; OCT, noctane; DET, n-decane; . OCA, 1-octanal; PES, pentanoic acid; HXS, hexanoic acid; H PS, heptanoic acid; OCS, octanoic acid; NOS, nonanoic acid.

Mapping the patterns to identified glomeruli. After physiological measurement, the brain sheath was digested with protease (Protease Type XIV, Sigma), and the AL stained with the dye RH 795 (M olecular Probes, Eugene, Oregon) for 30-45 minutes. Fluorescent photographs of the brain were then taken at different focal planes (interplane distance, 10 $\mu \mathrm{m})$. Photographs were contrast-enhanced using Adobe Photoshop, and borderlines of glomeruli reconstructed from the different focal depths, resulting in a glomerular map for each individual. With the aid of the computer-based atlas of the AL (www.neurobiologie.fu-berlin.de/honeybeeA Latlas ${ }^{20}$ ), single glomeruli could then be identified. To reduce overspill from adjacent glomeruli, the map of each glomerulus was eroded by 1 pixel. Response curves for all pixels corresponding to a glomerulus were then averaged for each stimulus. The corresponding response to the control air stimulus was then subtracted from each curve. Response intensity $\left(R_{\text {int }}\right)$ for that odor was integrated over ten frames following stimulus onset. As each odor was presented repeatedly, we took the median response as the response of that animal to that stimulus.

Statistical analysis: the olfactory code (Fig. 4). The response measurements give a vector, over identified glomeruli, for each odor and each animal. For each such vector, normalized responses were obtained by setting the strongest response $R_{\text {int }}$ at $100 \%$ and scaling other responses accordingly. For graphic representations in Figs. 2, 3 and 4, these values were categorized in five equal bins: 0-20\%, 20-40\%, 40-60\%, 60-80\% and $80-100 \%$. Noise was estimated as the average standard deviation of the normalized responses in frames 4-9 (that is, before stimulus). All responses within noise limits were omitted from the figures.

Both for odors and glomeruli, $n$ varied; not all glomeruli could be measured in all individuals-for instance, $n=21$ for 1-octanol, glomerulus T1-17 whereas $n=19$ for 1-octanol, glomerulus T1-24. A total of 14 glomeruli could be identified reliably in all individuals (marked in Figs. 3a and 4). To analyze the consistency range between individuals, we calculated the standard deviation on normalized responses for each glomerulus and for each odor. 


\section{ACKNOWLEDGEMENTS}

Thanks to J. Kunze and A. Gumbert for the odors HYA and NAR. Thanks to T. Faber, M. Wurm for comments on an earlier version of the manuscript. Supported by DFG M e365-21.

\section{ACCEPTED 8 M ARCH 1999}

1. Steward, W. B., Kauer, J. S. \& Shepherd, G. M . Functional organization of rat olfactory bulb analysed by the 2-deoxyglucose method. J. Comp. Neurol. 185, 715-734 (1979)

2. Cinelli, A. R., Hamilton, K. A. \& Kauer, J. S. Salamander olfactory bulb neuronal activity observed by video rate, voltage-sensitive dye imaging. III. Spatial and temporal properties of responses evoked by odorant stimulation. J. Neurophysiol. 73, 2053-2071 (1995).

3. Johnson, B. A. \& Leon, M. Spatial distribution of $\left[{ }^{14} \mathrm{C}\right] 2$-deoxyglucose uptake in the glomerular layer of the rat olfactory bulb following early odor preference learning. J. Comp N eurol. 376, 557-566 (1996)

4. Johnson, B. A., Woo, C. C.\& Leon, M. Spatial coding of odorant features in the glomerular layer of the rat olfactory bulb. J. Comp. Neurol. 393, 457-471 (1998).

5. Friedrich, R. W. \& Korsching, S. I. Combinatorial and chemotopic odorant coding in the zebrafish olfactory bulb visualized by optical imaging. Neuron 18, 737-752 (1997).

6. Friedrich, R. W. \& Korsching, S. I. Chemotopic, combinatorial, and noncombinatorial odorant representations in the olfactory bulb revealed using a voltage-sensitive axon tracer. J. N eurosci. 18, 9977-9988 (1998).

7. Rodrigues, V. Spatial coding of olfactory information in the antennal lobe of Drosophila melanogaster. Brain Res. 453, 299-307 (1988).

8. Distler, P. G., Bausenwein, B. \& Boeckh, J. Localization of odor-induced neuronal activity in the antennal lobes of the blowfly Calliphora vicina: a [3H ] 2-deoxyglucose labeling study. Brain Res. 805, 263-266 (1998)

9. Joerges, J., Kuettner, A., Galizia, C. G. \& M enzel, R. Representations of odors and odor mixtures visualized in the honeybee brain. Nature 387, 285-288 (1997).

10. Galizia, C. G., Joerges, J., Kuettner, A., Faber, T. \& M enzel, R. A semi-in-vivo preparation for optical recording of the insect brain. J. N eurosci. M ethods 76, 61-69 (1997).

11. Galizia, C. G., Nägler, K., Hölldobler, B. \& Menzel, R. Odor coding is bilaterally symmetrical in the antennal lobes of honeybees (Apis mellifera). Eur. J. Neurosci. 10, 2964-2974 (1998)

12. Faber, T., Joerges, J. \& Menzel, R. Associative learning modifies neural representations of odors in the insect brain. Nat. Neurosci. 2, 74-78 (1999).
13. Mombaerts, P. et al. Visualizing an olfactory sensory map. Cell $\mathbf{8 7 , 6 7 5 - 6 8 6}$ (1996).

14. Shepherd, G. M. Discrimination of molecular signals by the olfactory receptor neuron. Neuron 13, 771-790 (1994).

15. M ori, K., M ataga, N.\& Imamura, K. Differential specificities of single mitral cells in rabbit olfactory bulb for a homologous series of fatty acid odor molecules. J. Neurophysiol. 67, 786-789 (1992).

16. Teicher, M. H., Stewart, W. B., Kauer, J. S. \& Shepherd, G. M. Suckling pheromone stimulation of a modified glomerular region in the developing rat olfactory bulb revealed by the 2-deoxyglucose method. Brain Res. 194, 530-535 (1980).

17. Hildebrand, J. G. Olfactory control of behavior in moths: Central processing of odor information and the functional significance of olfactory glomeruli. J. Comp. Physiol. A 178, 5-19 (1996).

18. Rospars, J. P. Structure and development of the insect antennodeutocerebral system. Int. J. Insect M orphol. Embryol. 17, 243-294 (1988).

19. Flanagan, D. \& M ercer, A. R. An atlas and 3-D reconstruction of the antennal lobes in the worker honey bee, A pis mellifera L. (Hymenoptera: Apidae). Int. J. Insect M orphol. Embryol. 18, 145-159 (1989).

20. Galizia, C. G., M cl lwrath, S. L. \& M enzel, R. A digital three-dimensional atlas of the honeybee antennal lobe based on optical sections acquired using confocal microscopy. Cell Tissue Res. 295, 383-394 (1999).

21. Stopfer, M., Bhagavan, S., Smith, B. H. \& Laurent, G. Impaired odor discrimination on desynchronization of odor-encoding neural assemblies. Nature 390, 70-74 (1997).

22. Kramer, E. The orientation of walking honeybees in odor fields with small concentration gradients. Physiol. Entomol. 27-37 (1976)

23. Bhagavan, S. \& Smith, B. H. Olfactory conditioning in the honey bee, Apis mellifera: Effects of odor intensity. Physiol. Behav. 61, 107-117 (1997)

24. Slotnick, B. M., Graham, S., Laing, D. G. \& Bell, G. A. Odor detection in rats with lesions of areas identified using the 2-deoxyglucose method. Ann. NY Acad. Sci. 510, 630-631 (1987).

25. Lu, X.-C. M . \& Slotnick, B. M. Olfaction in rats with extensive lesions of the olfactory bulbs: implications for odor coding. Neuroscience 984, 849-866 (1998).

26. Sigg, D., Thompson, C. M . \& M ercer, A. R. Activity-dependent changes to the brain and behavior of the honey bee, Apis mellifera (L.). J. Neurosci. 17, 7148-7156 (1997).

27. Winnington, A. P., Napper, R. M. \& Mercer, A. R. Structural plasticity of identified glomeruli in the antennal lobes of the adult worker honey bee. J. Comp. Neurol. 365, 479-490 (1996).

28. Burrows, M. The Neurobiology of an Insect Brain. (Oxford Univ. Press, Oxford, 1996)

29. Yokoi, M., M ori, K. \& Nakanishi, S. Refinement of odor molecule tuning by dendrodendritic synaptic inhibition in the olfactory bulb. Proc. Natl. Acad. Sci. USA 92, 3371-3375 (1995). 